\title{
PROCESOS DE MANEJO COSTERO INTEGRADO EN ECUADOR Y SRI LANKA: UNA PERSPECTIVA DE COMPARACIÓN
}

\author{
INTEGRATED COASTAL ZONE MANAGEMENT PROCESS IN ECUADOR AND \\ SRI LANKA: A COMPARATIVE PERSPECTIVE
}

Marcela de Avellar-Mascarello, Martin García-Cartagena y Eddy Jara-Torres

\begin{abstract}
RESUMEN
Ecuador (en 1989) y Sri Lanka (en 1990) empezaron sus procesos de Manejo Costero Integrado - MCI utilizando fondos de la misma Cooperación Internacional y asesoramiento y evaluación de la misma universidad extranjera. Tomando en cuenta estas similitudes y que ambos son países tropicales en vía de desarrollo se efectuó un análisis comparativo entre ambos. A través del modelo conceptual de ciclos de MCI sugerido por GESAMP y una amplia revisión bibliográfica y documental sobre la temática, fueron analizados ambos procesos de $\mathrm{MCI}$, a fin de entender sus fortalezas y debilidades para fomentar nuevos procesos de MCI, así como también evaluar el uso del modelo conceptual de GESAMP como herramienta de análisis. Lo que en principio parecían procesos similares, en la práctica fueron muy distintos. Ecuador presentó un proceso más orientado a la participación desde el comienzo, mientras Sri Lanka solamente en la tercera generación logró integrar aproximaciones participativas. De otro lado, Sri Lanka tuvo un proceso más ordenado y centrado en desarrollo institucional, estableciendo primero un marco institucional que sirviera de base al comienzo del proceso de MCI. Ambos países han alcanzado resultados de primer orden (cambios institucionales), de segundo orden (cambios en el comportamiento) y posiblemente de tercer orden (cambios socioeconómicos y ambientales). Se concluye con este estudio de caso que los procesos participativos, la voluntad política, los procesos de evaluación y la financiación fueron fundamentales para el éxito de ambos proceso de MCI. También se encontró que el modelo conceptual propuesto por GESAMP representa una herramienta teórica fundamental para la planificación y análisis de los procesos de MCI, pero podría ser revisado y contrapuesto empíricamente a través de una investigación más profunda, con un mayor número de casos internacionales concretos de implementación de planes de MCI.
\end{abstract}

PALABRAS CLAVE: Cooperación internacional, costa, evaluación de programas, manejo costero integrado, Ecuador, Sri Lanka

\begin{abstract}
Ecuador (1989) and Sri Lanka (1990) began their Integrated Coastal Zone Management (ICZM) Processes through the same International Cooperation funds, counseling and evaluation. Taking these facts into account, we developed a comparative analysis of their Integrated Coastal Zone Management processes. Using the GESAMP ICZM cycle conceptual framework, and through an extensive literature and document review on the subject, both processes were analyzed in order to determine their strengths and weaknesses and evaluate the GESAMP cycle conceptual framework as an analysis tool. What appeared to be similar processes, turned out to be very different. Ecuador showed a more participatory approach towards ICZM plan design and implementation from the beginning, while Sri Lanka only implemented this approach in its third ICZM generation plan. On the other hand, Sri Lanka had a more institutional program, thus generating from the outset a better and more organized institutional framework for ICZM than Ecuador. Both countries have achieved first-order results (institutional changes), second-order ones (behavioral changes), and possibly third-order ones too (socio-ecological indicator improvements). We conclude through this paper that participatory approaches, political will, effective evaluation processes and international aid are fundamental elements for successful ICZM processes. We also conclude that the conceptual framework proposed by GESAMP is a very important theoretical analysis tool through which ICZM processes can be analyzed. But this theoretical tool should be revised and tested through empirically based analysis on other international ICZM application study cases.
\end{abstract}

KEY WORDS: International Cooperation, coast, program evaluation, integrated coastal management, Ecuador, Sri Lanka

Licenciada en Oceanografía, maestranda en Manejo Costero Integrado, en el Centro Interdisciplinario para el Manejo Costero Integrado del Cono Sur- Universidad de la República- Maldonado- Uruguay; E-mail: mascarellomarcela@gmail.com (M.A.M.). Licenciado en Relaciones Internacionales, maestrando en Manejo Costero Integrado, en el Centro Interdisciplinario para el Manejo Costero Integrado del Cono Sur- Universidad de la República- Maldonado- Uruguay; E-mail: martingarciacartagena@gmail.com (M.G.C.). Licenciado en Biología, maestrando en Manejo Costero Integrado, en el Centro Interdisciplinario para el Manejo Costero Integrado del Cono Sur- Universidad de la República- Maldonado- Uruguay; E-mail: eddie_jara_t@hotmail.com (E.J.T.) 


\section{INTRODUCTION}

A partir de finales de los años sesenta comenzó a surgir la necesidad de generar algún tipo de control ambiental y de medidas que incentivaran la responsabilidad socioambiental. La Conferencia de las Naciones Unidas para el Medio Ambiente Humano desarrollada en Estocolmo (ONU,1972), junto con la Adopción de la Convención de las Naciones Unidas sobre el Derecho del Mar (ONU, 1982), sentaron las bases para que algunas agencias internacionales decidieran financiar proyectos pilotos de Manejo Costero Integrado (MCI) en países en desarrollo.

Durante la década del 80 muchos gobiernos implementaron dentro de sus agendas el concepto de manejo sustentable de los recursos naturales, desarrollando a la par algunas propuestas de programas para el manejo costero con financiamiento de cooperación internacional. El concepto de manejo costero integrado se consolidó teórica y prácticamente a partir de la adopción de la Agenda XXI en la Conferencia de las Naciones Unidas sobre el Medio Ambiente y Desarrollo llevada a cabo en Rio de Janeiro (PNUMA, 1992). Éste es definido como un proceso continuo y dinámico en el cual decisiones son tomadas para el uso sostenible, desarrollo y protección de zonas costeras y marinas, así como de sus recursos (Knecht y Archer, 1993; Sorensen, 1993; World Bank, 1993; GESAMP, 1996 y 1999; Cicin-Sain y Knecht, 1998; Vallega, 1999; Granados, 2012). Hildebrand (2002) enfatizó la necesidad de integrar gobierno, comunidad, sector privado y ciencia para la elaboración e implementación de los programas de MCI.

Bajo este contexto, surge en 1983 el Programa Internacional de Manejo de los Recursos Costeros, auspiciado por la Agencia para el Desarrollo Internacional de Estados Unidos (USAID). Este proyecto fue implementado en tres países: Ecuador, Sri Lanka y Tailandia. De esta manera, Ecuador y Sri Lanka, en 1989 y 1990 respectivamente, comenzaron a desarrollar políticas de manejo costero con el asesoramiento de la Universidad de Rhode Island (URI) (Lowry y Wickremeratne, 1989; Ochoa, 1995; Barragán, 2001; Coello, 2008).

La Republica del Ecuador, se localiza en América del Sur, con su costa proyectada al Océano Pacífico. Se halla entre las latitudes $1^{\circ} 27^{\prime} \mathrm{N}$ y $5^{\circ} 0^{\prime} \mathrm{S}$, y las longitudes $75^{\circ} 11^{\prime}$ y $81^{\circ} 0$ 'O. Posee una costa de $68.038 .007,78$ $\mathrm{km}^{2}$ y cuenta con 79 cuencas fluviales e innumerables ecosistemas asociados que resultan en elevados índices de biodiversidad. Su economía está basada en sus recursos naturales renovables y no renovables, con énfasis en industria del petróleo, agricultura, industria, pesca, camaroneras, turismo y minerales (Arriaga et al., 1999; Arriaga, 2000).

Sri Lanka, es un archipiélago formado por una gran isla volcánica y otras pequeñas islas a su alrededor. Ubicada en el continente asiático, en el Océano Índico, se sitúa en el hemisferio norte entre las latitudes $5^{\circ} 58^{\prime}$ y $9^{\circ} 48^{\prime}$ y al este del meridiano de Greenwich entre las longitudes de $79^{\circ} 41^{\prime}$ y $81^{\circ} 52^{\prime}$. Al igual que Ecuador, Sri Lanka posee una economía basada en sus sistemas agroecológicos. Entre las principales actividades económicas costeras se pueden destacar las siguientes: venta de bienes al consumidor, industria, transporte, comunicaciones, agricultura, forestación, pesca, construcción y minería, con una alta concentración de minerales en la zona suroeste y noreste de sus costas (Savundranayagam et al., 1994).

Es importante destacar que a pesar de los distintos desafíos que el $\mathrm{MCI}$ viene atravesando en los últimos 40 años y sus diversas debilidades, se ha mostrado como uno de los marcos organizacionales más efectivos para lograr metas de largo plazo en desarrollo o conservación, y ha confirmado su efectividad como una metodología que contribuye al desarrollo sustentable (Olsen et al., 2004), disminuyendo el riesgo de inversiones, ordenando los esfuerzos de desarrollo, minimizando los conflictos entre los diferentes usos, fortaleciendo la participación y democracia local y desarrollando la responsabilidad social (Ochoa, 2001). Olsen y Ochoa (2007) resaltan que "un buen caso de estudio tiene alto potencial para aportar evidencias de cómo opera el sistema de gobernanza de los usos en los ecosistemas a lo largo a veces de décadas".

En esto contexto, son diversas las publicaciones que han buscado extraer enseñanzas a partir de experiencias de MCI (Cicin-Sain y Knecht, 1998; Olsen et al., 1997; Rodríguez y Windevoxhel, 1998; Lowry et al., 1999a; Ochoa, 2001; Ochoa et al., 2001; Polette y Silva, 2003; 
Olsen et al., 2004; Stojanovic et al., 2004; Cummins et al., 2004; Olsen y Ochoa, 2007; Tagliani y Asmus, 2011; Gonzáles et al., 2012), a fin de constatar las debilidades y fortalezas de los procesos con la voluntad de mejorar y adaptar la implementación de políticas públicas orientadas a un manejo sostenible de las costas, considerando los aspectos sociales, económicos y ambientales de forma integrada y equitativa.

Fundamentados en estos antecedentes y teniendo en cuenta que Ecuador y Sri Lanka presentan características agroecológicas propias de los trópicos, basadas principalmente en los recursos pesqueros, y que comenzaron sus procesos de manejo costero simultáneamente y bajo un mismo programa de cooperación internacional, en el presente trabajo se desarrolla un análisis comparativo a partir de las fortalezas y debilidades de cada proceso, que a su vez pueda servir como un modelo para futuros procesos de implementación (Molina, 2000; Coast Conservation Department- Sri Lanka, 2004).

\section{METODOLOGÍA}

Este trabajo se llevó a cabo a través de un análisis documental y bibliográfico. Se seleccionaron documentos públicos directamente relacionados con los procesos de MCI de ambos países. Entre los documentos que se revisaron se encuentran los planes de manejo costero de ambos países en todas sus etapas, así como artículos relacionados con sus procesos de planificación, implementación, ejecución y decretos y leyes que surgieron de sus planes (Tabla 1).

Para analizar los Programas de Manejo Costero integrado (PMCI) se utilizaron las etapas definidas en el modelo conceptual de ciclos propuesto por GESAMP (1996), en el que se valoró la primera generación de los dos países, para evaluar cada etapa por separado y así facilitar la comprensión y comparación entre los distintos procesos. Las generaciones siguientes fueron analizadas de forma integral, teniendo en cuenta que su desarrollo se relaciona directamente con los logros y debilidades de la generación anterior, dificultando la sistematización de los datos por fases.

\section{Fases de un Programa de MCI}

El modelo para el proceso de MCI propuesto por GESAMP (1996) sirve como una guía tanto para quien está implementando el proceso como para quién lo evalúa, haciendo posible organizar la información y las etapas de desarrollo de forma didáctica y práctica.

El modelo propuesto está dividido en generaciones formadas por cinco fases (no obligatorias pero ideales): identificación y evaluación de asuntos claves, preparación del programa, adopción formal y financiamiento, implementación y, por último, evaluación. Con el cumplimiento de estas cinco fases se considera concluida una generación y comienza una nueva, de haberla. Siguiendo los conceptos del modelo, el desarrollo de una segunda generación debería tomar en consideración los resultados de la última fase (fase de evaluación) de la generación anterior. Dicho de otra forma, las fortalezas y debilidades constatadas en el proceso de evaluación de la quinta fase deberían marcar el diseño de la siguiente generación.

\section{Fase 1. Identificación y evaluación de asuntos claves}

En la fase de identificación y evaluación de asuntos claves surge la demanda de la implementación del proceso de MCI. A partir de esta demanda, surgen los asuntos claves y las principales preguntas que deben ser abordadas: ¿En qué condición se encuentran los ecosistemas costeros? ¿Cuál es el contexto político/ institucional? ¿Cuál es el contexto de desarrollo? ¿Cuáles son los vacíos en el conocimiento científico? A partir de estas preguntas, un equipo multi, inter o transdisciplinar debe preparar un documento que describa los asuntos sobre los cuales se enfocará el programa y los valores, políticas o restricciones bajo los cuales operará.

El mismo documento debe identificar las implicaciones de corto y largo plazo de las tendencias existentes y sugerir prioridades para la acción. Es importante destacar que este tipo de documento constituye la base para consultas sobre las metas y prioridades del programa, entre manejadores, científicos y público. 
Tabla 1. Principales fuentes de información para la conducción del análisis

\begin{tabular}{|c|c|c|c|}
\hline País & Nombre del documento & Año de publicación & Tipo de documento \\
\hline \multirow{13}{*}{ Ecuador } & $\begin{array}{l}\text { Ordenación y desarrollo integral de las zonas costeras: informe del } \\
\text { seminario realizado por el gobierno del Ecuador conjuntamente con } \\
\text { las Naciones Unidas }\end{array}$ & 1983 & Informe \\
\hline & Decreto $n^{0} 375$ & 1989 & Normativa \\
\hline & Decreto n ${ }^{0} 3399$ & 1992 & Normativa \\
\hline & Decreto $n^{0} 2451$ & 1995 & Normativa \\
\hline & Manejo costero integrado en Ecuador & 1995 & Plan \\
\hline & Principios relevantes para la construcción del PMRC en Ecuador & 1995 & Publicación \\
\hline & Manejo Costero Integrado (MCI) del Ecuador & 2000 & Publicación \\
\hline & Manejo de la pesquería de post-larva de camarón. & 2000 & Informe \\
\hline & $\begin{array}{l}\text { The common methodology for learning: Ecuador's Pioneering Initiative } \\
\text { in Integrated Coastal Management }\end{array}$ & 2000 & Publicación \\
\hline & $\begin{array}{l}\text { Experiencias de gestión integrada de zonas costeras en Ecuador y } \\
\text { Centroamérica }\end{array}$ & 2001 & Publicación \\
\hline & Decreto $n^{\circ} 1254$ & 2008 & Normativa \\
\hline & $\begin{array}{l}\text { Reglamento Operativo del Proyecto de Manejo de Recursos Costeros- } \\
\text { Etapa II (PMRC II). }\end{array}$ & 2009 & Normativa \\
\hline & $\begin{array}{l}\text { Propuesta de: Política Costera del Ecuador (Costas vivas y saludables } \\
\text { para el Desarrollo Nacional) }\end{array}$ & 2010 & Publicación \\
\hline \multirow{10}{*}{ Sri Lanka } & Coast Conservation Act No. 57 of 1981 & 1981 & Normativa \\
\hline & Coastal Area Management in Sri Lanka & 1989 & Publicación \\
\hline & Coastal Zone Management Plan & 1990 & Plan \\
\hline & $\begin{array}{l}\text { Coastal 2000: Recommendations for A Resource Management Strategy } \\
\text { for Sri Lanka's Coastal Region, Volumes I and II }\end{array}$ & 1992 & Informe \\
\hline & Coastal Management in Sri Lanka & 1993 & Publicación \\
\hline & The Economic Significance of the Coastal Region of Sri Lanka & 1994 & Informe \\
\hline & $\begin{array}{l}\text { Between a coral reef and a hard place: The politics of coastal } \\
\text { management in Sri Lanka }\end{array}$ & 1995 & Publicación \\
\hline & Revised Coastal Zone Management Plan Sri Lanka & 1997 & Plan \\
\hline & $\begin{array}{l}\text { Policy-relevant assessment of community-level coastal management } \\
\text { projects in Sri Lanka }\end{array}$ & 1999 & Publicación \\
\hline & Second Revision of Coastal Zone Management Plan Sri Lanka & 2004 & Informe \\
\hline
\end{tabular}

\section{Fase 2. Preparación del Programa}

Proceso prolongado de consulta y planificación, donde se definen claramente los objetivos específicos del programa. Debe contar con amplia participación buscando que los objetivos reflejen las aspiraciones y valores de quienes tienen interés en las áreas y recursos a ser manejados. Es un proceso reiterativo, que incluye análisis, debates e implementación a escala piloto, que posee como objetivo principal la consolidación de un 
Plan de Manejo con "visión del futuro” y que exprese, en términos reales y tangibles, la calidad ambiental.

El rol de demandas del equipo de MCI y científicos involucrados es el de contestar preguntas como: ¿Cuál el peso relativo de los factores antropogénicos y naturales en los cambios particulares de los sistemas y recursos costeros? ¿Cuáles son las consecuencias y beneficios sociales y económicos de corto y largo plazo de acuerdo a las tendencias de los usos de los ambientes costeros? Finalmente, en esta fase se deben proponer estrategias y mecanismos para mitigar y revertir la degradación ambiental y estimar los costos y beneficios sociales, ambientales y económicos de cursos de acción alternativos.

\section{Fase 3. Adopción formal y financiamiento}

La adopción formal de un programa requiere una decisión administrativa normalmente de alto nivel (del líder de una institución gubernamental, de un ministro del gabinete o aún de una firma presidencial). La decisión depende de consideraciones y acuerdos sobre fondos asignados para cada fase del programa. Los fondos asignados puede ser de diversos orígenes, como por ejemplo: fuentes nacionales públicas, como pueden ser instituciones estatales de la más diversa índole y que probablemente tengan competencias en las zonas costeras; fuentes internacionales públicas, como pueden ser agencias bilaterales de cooperación para el desarrollo o programas multilaterales de organismos internacionales; y finalmente fuentes de carácter mixtos, que involucran tanto fondos internacionales como nacionales públicos y también privados, en forma de donaciones bajo conceptos de responsabilidad civil, entre otros.

\section{Fase 4. Implementación}

Fase operacional, en la cual el énfasis gira en torno a la introducción de nuevas formas de desarrollo y de uso del recurso, hacia nuevos arreglos institucionales y sistemas de monitoreo y hacia la aplicación de nuevos controles, regulaciones e incentivos. Las actividades prioritarias contemplan resolución de conflictos, monitoreo, aplicación de indicadores de eficacia, coordinación interinstitucional, construcción de infraestructura, acciones de desarrollo, educación pública, capacitación de personal, planificación e investigación en nuevas áreas o problemas.

\section{Fase 5. Evaluación}

Esta es la fase de mayor aprendizaje, pero es también muchas veces omitida en el proceso o realizada de forma superficial o incluso con carácter confidencial sin hacer públicos los resultados. Ella debe ser la articulación crítica entre una generación y otra, respondiendo dos preguntas muy amplias: ¿Qué se ha alcanzado y aprendido de la generación precedente y cómo debería este aprendizaje afectar el diseño y enfoque de la siguiente generación? ¿Cómo ha cambiado el contexto (asuntos claves, manejo ambiental) desde que se inició el programa?

\section{Sistematización de la información compilada según fases definidas en GESAMP (1996)}

Considerando las bibliografías sistematizadas en la Tabla 1 y el modelo propuesto por GESAMP (1996) descrito anteriormente, se realizó el ejercicio de sistematizar la información compilada a partir del modelo. Esta forma de sistematización de la información fue aplicada a la primera generación de ambos países, pero no para las generaciones siguientes, ya que estas últimas surgen de la evaluación de la primera y no se encontró en formato de acceso público suficiente información para llegar a describirlas por fases.

\section{RESULTADOS}

\section{Ecuador}

Se lograron identificar dos generaciones de MCI llevadas a cabo por el gobierno ecuatoriano, asistido por diversos actores internacionales tanto en aportes técnicos como de financiamiento. Estas generaciones son descritas a continuación y resumidas en la Figura 1.

\section{Primera Generación (1986-2000)}

- Fase 1. Identificación y evaluación de asuntos claves

En Ecuador los aprendizajes sobre el MCI empezaron a finales de los años 80, a poco tiempo de haber salido de las dictaduras y de los conflictos internos, que separaron la población en bandos opuestos. En una sociedad civil marginalizada de la participación en los beneficios de los éxitos económicos, se empezó a levantar la bandera del manejo costero (Ochoa, 2001). 


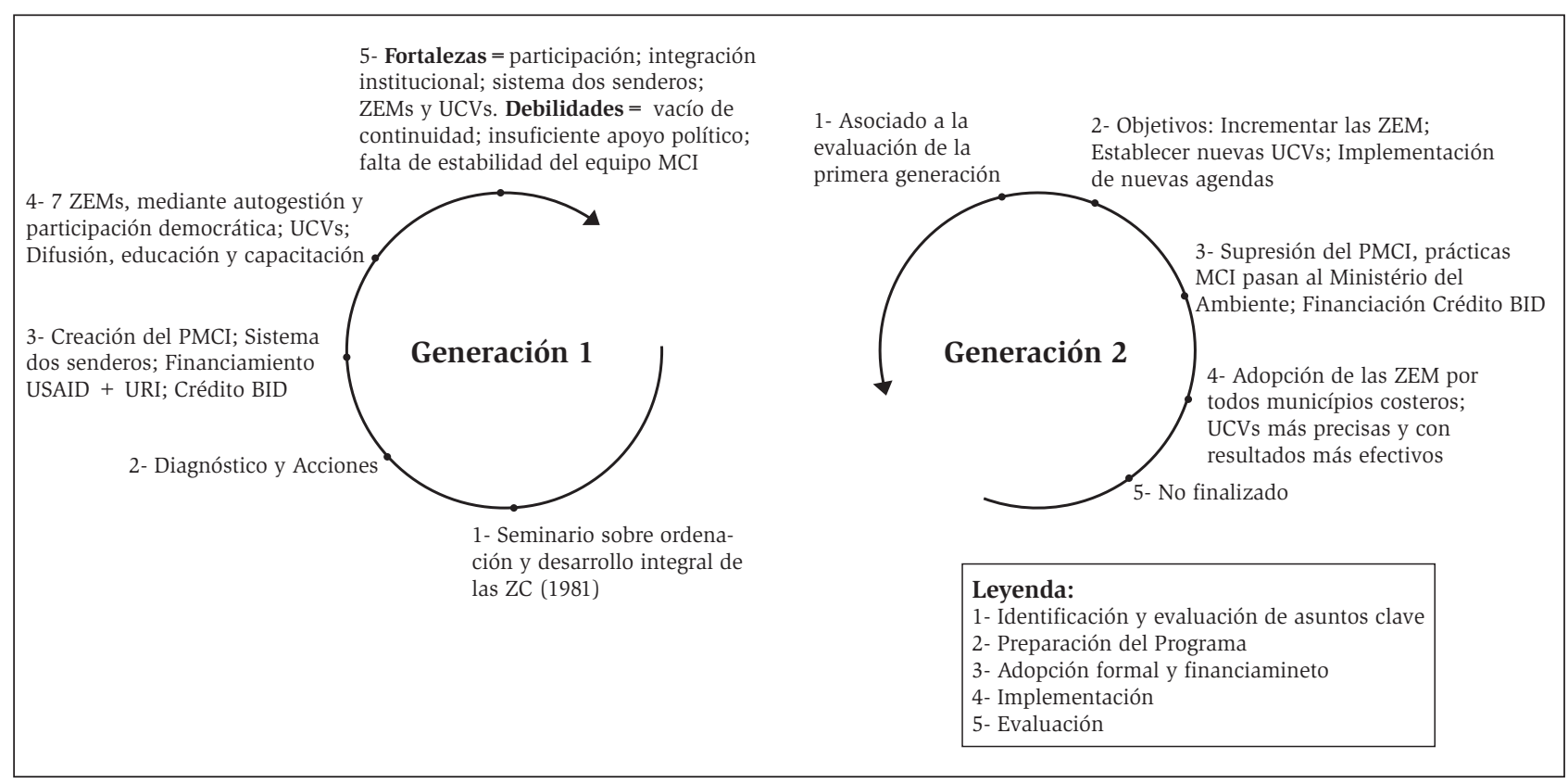

Figura 1. Esquema representando el proceso de Manejo Costero Integrado de Ecuador siguiendo el modelo de análisis propuesto por GESAMP (1996).En la figura las siglas ZC corresponden a Zona(s) Costera(s); PMCI a Programa de Manejo Costero Integrado; USAID a la Agencia para el Desarrollo Internacional de Estados Unidos; URI a la Universidad de Rhode Island; BID al Banco Interamericano de Desarrollo; ZEM a las Zonas Especiales de Manejo; UCV a las Unidades de Conservación y Vigilancia; MCI al Manejo Costero Integrado.

En 1981, Ecuador celebró el "Seminario sobre ordenación y desarrollo integral de las zonas costeras", que fue auspiciado por Naciones Unidas y la Armada Nacional de Ecuador; con la participación de técnicos nacionales e internacionales. Esto proporcionó una primera visión integrada de los conocimientos sobre la zona costera del país y sembró la semilla del MCI, al identificar ciertas necesidades que se hacían urgentes de abordar (Armada del Ecuador, 1983). Se podría mencionar que algunas de estas necesidades fueron: "formulación, ejecución y control de una política de desarrollo y ordenación integral de las zonas costeras", "necesidad de un órgano gubernamental de asuntos costeros", promover mayores controles sobre las actividades que se desarrollan y que impactan sobre la costa, relevamiento de la zona costera en general (inventarios, identificación de zonas de particular riesgo, mapas), y finalmente, se identificó la falta de investigación en la temática, como una problemática a ser abordada en la brevedad 48 (Arriaga, 2000). Estas fueron las bases que sirvieron de pilar para el establecimiento del primer Convenio que Ecuador firmó el 3 de marzo de 1986, con la USAID y la URI (Arriaga, 2000).

\section{- Fase 2. Preparación del Programa}

A través del Convenio mencionado se establecieron ciertas acciones orientadas a promover el desarrollo de la región costera del Ecuador y el uso sustentable de los recursos. Estas acciones fueron diseñadas para desarrollar los siguientes puntos; la elaboración del Programa de Manejo de Recursos Costeros (PMRC), el análisis de un marco institucional y legal del Manejo de Recursos Costeros (MRC), la recolección y difusión de la información existente, elaboración de perfiles de las regiones costeras del Ecuador, elaboración de sistemas de clasificación de usos de aguas y tierras costeras, el desarrollo del litoral bajo normas de protección, relevamiento de los impactos sociales y ecosistémicos de la cría del camarón en Ecuador y el desarrollo de observaciones periódicas. Olsen et al. (1995) también resaltan como acciones el desarrollo de programas de educación pública para la concientización sobre los problemas y el valor de la costa y sus recursos, la protección y recuperación de algunos ecosistemas naturales y el ordenamiento territorial, entre otras. 
Es importante destacar que el proceso de diagnóstico acerca de los asuntos claves de manejo, la selección de áreas de manejo y la determinación de las características institucionales del PMRC fueron productos del consenso obtenido en los foros abiertos. En estas instancias abiertas se constató la participación de líderes y personalidades destacadas de las provincias, así como de autoridades municipales y organizaciones regionales de desarrollo, agrupaciones de artesanos que dependen de los recursos costeros, entidades académicas y ONG's (Arriaga, 2000).

- Fase 3. Adopción formal y financiamiento

El proceso de organización y preparación del PMRCEcuador se desarrolló a través de dos etapas de financiamiento. La primera (1986 a 1994) a través de USAID y conducida por la URI, que gestionaron el diseño del programa, la capacitación del personal y la planificación del MCI en Zonas Específicas de Manejo (ZEM), es decir, fue la fase de preparación del programa. La segunda, destinada a la implementación del programa mediante un crédito del Banco Interamericano de Desarrollo (BID) e iniciada en 1996, finalizó con la implementación, con lo cual se cerró el ciclo de la primera generación del MCI en Ecuador (Ochoa, 1995).

La figura institucional creada fue el PMRC y funcionaba como un órgano del Estado con cierto grado de autonomía. La función principal de esta institución era estructurar y coordinar a las distintas autoridades nacionales que tuviesen influencia y competencia en la zona costera. También estaba a cargo de la coordinación entre los lineamientos nacionales del plan de manejo, y los planes y acciones desarrolladas a nivel local. La estructura y estrategia operativa obedecen a la necesidad de funcionamiento en dos senderos o niveles: (a) en el nivel del gobierno central, donde se adoptan las políticas y estrategias nacionales (autoridades de alto nivel que orientan y controlan el MCI a nivel nacional, y que definen las políticas globales); y (b) en el nivel local y franja costera, donde se realiza el ejercicio práctico del MCI, reuniendo dos aspectos: Planificación y Gestión costera (Arriaga, 2000).

Posterior a estos procesos, se hace una aprobación formal del PMRC mediante el Decreto Ejecutivo número 375, Registro Oficial No. 117 de 26 de enero de 1989 (Ecuador, 1989). Este decreto fue sustituido por el D.E.
3399 (Ecuador, 1992) y este, a su vez, por el D.E. 2451 (Ecuador, 1995 1995).

\section{- Fase 4. Implementación}

Se desarrollaron siete ZEMs, mediante autogestión y participación democrática (Ochoa, 1995), ejercicios prácticos de manejo (EP) (pequeños proyectos seleccionados por grupos de usuarios o comunidades y aprobados por el Comité de la ZEM) (Arriaga, 2000), Unidades de Conservación y Vigilancia (UCV), órganos de apoyo y coordinación para una gestión de recursos enfocada en los ecosistemas costeros, cuyo objetivo principal es la "protección, conservación y buen uso de los recursos costeros", así como la resolución de conflictos (Olsen et al., 1995). En este programa la difusión, educación y capacitación fueron indispensables. El énfasis de este plan estuvo puesto en el habitante costero, y, esencialmente, en los usuarios directos de los recursos, sean estos los tradicionales 0 los empresarios recientes (Ochoa, 1995). En la Tabla 2 es posible observar algunos de los principales proyectos desarrollados con participación de la población.

\section{• Fase 5. Evaluación}

Un resultado clave derivado de la experiencia positiva del PMRC fue el hecho de que los principios, estrategias y estructura institucional mantuvieran su validez con procesos claramente participativos desde un comienzo, obteniéndose resultados muy prácticos, como la integración de autoridades para la gestión de los recursos, lo que constituyó un mecanismo adecuado para la gestión ambiental enfocada en ecosistemas. La estrategia de los "dos senderos" del PMRC integra muy bien la gestión nacional y local, permitiendo una adecuación a un sistema de descentralización y autogestión de las áreas costeras, sin intervenir los intereses de los gobiernos de turno (Arriaga, 2000). Como estableció Ochoa (2001): "El gran acierto fue diseñar el PMRC como un autodesafío, como un esfuerzo conjunto de beneficiarios, planificadores y ejecutores que ven en la colaboración la fuente y la vía de ventajas y ganancias".

Los principales problemas que se presentaron se derivaron del vacío de continuidad de las actividades iniciadas, del debilitamiento de las estructuras y prácticas participativas que operan en las ZEM, del insuficiente apoyo político al MCI y de la falta de estabilidad políticoadministrativa en el equipo guía del proceso. 
Tabla 2. Principales proyectos realizados con participación local en la primera generación del PMRC de Ecuador

\begin{tabular}{ll}
\hline \multicolumn{1}{c}{ Acciones } & \multicolumn{1}{c}{ Proyectos } \\
\hline Manejo de manglares & $\begin{array}{l}\text { Proyectos de reforestación y conservación del manglar, } \\
\text { implementación de las Unidades de Conservación y Vigilancia. }\end{array}$ \\
Ordenamiento costero y turismo & $\begin{array}{l}\text { Planes de Ordenamiento para las playas de las Zonas Específicas } \\
\text { de Manejo (ZEM) }\end{array}$ \\
Servicios Básicos y Saneamiento Ambiental & $\begin{array}{l}\text { Agua potable, letrinas, recolección y disposición final de basura; } \\
\text { Infraestructura y servicios para la pesca artesanal, estudio y manejo } \\
\text { de pescarías críticas, experiencias con ZEM en la “autoveda”, } \\
\text { reducción de impacto en las especies que acompañan los camarones } \\
\text { en la recolección de portlarvas. Elaboración de proyectos pilotos } \\
\text { de cría de conchas de manglar, y capacitación de los “larveros” en } \\
\text { identificación y cuantificación de postlarvas. }\end{array}$ \\
\hline
\end{tabular}

Fuente: Autores, basado en Ochoa (1995).

\section{Segunda Generación (2004-hasta la actualidad)}

La Unidad Ejecutora con sede en Guayaquil, adscrita a la Presidencia de la República mediante el Crédito BID 1531 OC/EC firmado el 26 de octubre de 2004, establece la segunda generación para el PMRC. El principal objetivo que se planteó fue incrementar las ZEM, logrando ser adoptadas por todos los municipios costeros. También se planteó instalar nuevas UVC, desarrollándose de una manera más precisa y con mejores resultados. Junto a estas acciones, se plantearon nuevas estrategias con la implementación de agendas y ordenanzas municipales enfocadas al PMRC y al MCI, las cuales, a su vez, ayudaban a gestionar y conseguir fondos para proyectos amigables con la naturaleza (Olsen, 2000).

El 12 de Agosto del 2008 se expidió el Decreto Ejecutivo No. 1254 (Ecuador, 2008), en cuyo Artículo 1 se decide la Supresión del PMRC, debido a que su existencia dejó de ser indispensable para el desarrollo de las áreas costeras del país. En el Artículo 2 se indica que todas las competencias, atribuciones, funciones, representaciones y delegaciones, constantes en leyes, reglamentos y demás instrumentos normativos, que hasta esta fecha eran ejercidas por el PMRC, pasarán a ser ejercidas en bloque por el Ministerio del Ambiente. Mediante el Acuerdo No. 025, de fecha 10 de marzo del 2009, se expide el Reglamento Operativo del PMRC II (Ecuador, 2009).
Las siguientes acciones que Ecuador tomaría orientadas al manejo de los recursos costeros fueron guiadas por una serie de lineamientos estratégicos enumerados en una propuesta de Política Costera de Ecuador, del año 2008. A través de estos lineamientos el Estado Ecuatoriano declaró de interés nacional la gestión sustentable e integrada de la zona costera y que todas las acciones que se ejecuten en zonas costeras serán enmarcadas en el concepto de Desarrollo Sustentable. Como objetivos concretos se plantearon los siguientes: reducir la pobreza en zonas costeras; generar un marco normativo e institucional integral; asegurar la conservación de la biodiversidad costera a través de la ampliación de las áreas marinas y costeras protegidas; incentivar el desarrollo de actividades productivas competitivas, sustentables y responsables, en especial pesca, acuacultura, agricultura, turismo y actividades mineras e hidrocarburíferas; promover la valorización de la diversa herencia costera y fortalecer la educación, la formación profesional, la capacitación y la participación ciudadana; dar prioridad a la investigación básica y aplicada de los recursos y procesos naturales y sociales de la zona costera; fortalecer la gestión de riesgos en la zona costera; y finalmente tomar las medidas pertinentes para honrar los compromisos establecidos en los instrumentos regionales e internacionales y colaborar activamente en la agenda internacional relativa a gestión de los recursos costeros (Coello, 2008). 


\section{Sri Lanka}

Se lograron identificar tres generaciones de MCI llevadas a cabo por el gobierno de Sri Lanka, asistido también por diversos actores internacionales, tanto en aportes técnicos como de financiamiento. Estas generaciones son descriptas a continuación y resumidas en la Figura 2.

El MCI en Sri Lanka empieza con la creación del CCA (Coast Conservation Act) - Act $\mathrm{N}^{\circ} 57$, elaborado en 1981 (Sri Lanka, 1981) y sancionado en 1983. Esta ley estableció los pilares institucionales sobre los cuales Sri Lanka construiría su proceso de MCI. Los principales aportes de esta ley fueron:

- Creación del Coast Conservation Department (CCD).

- Creación del Coast Conservation Advisory Council (CCAC).

- Definición de una Zona Costera (ZC).

- Sistema de permisos para llevar a cabo actividades que impacten en la ZC.

- Encomendación al CCD de elaborar el primer CZMP (Coastal Zone Management Plan) para Sri Lanka.

\section{Primera Generación (1990 - 1997)}

- Fase 1. Identificación y evaluación de asuntos claves

La motivación para el desarrollo del CZMP de Sri Lanka se origina en las propuestas e incentivos internacionales sumados a una necesidad y voluntad interna de arreglos y mejorías frente a problemas socioambientales que se venían constatando en ese país. Los problemas más evidentes detectados fueron erosión, conflictos de acceso a la faja costera, depredación y degradación de hábitats y recursos costeros, riesgo y vulnerabilidad a eventos de tsunamis y falta de facilidades para los visitantes, entre otros (Coast Conservation Department, 1990).

Las herramientas de diagnóstico utilizadas para identificar las distintas problemáticas costeras fueron talleres, reuniones técnicas con especialistas, entrevistas individuales con especialistas y finalmente distintos tipos de información sobre uso de suelos. Queda claro que estos primeros pasos que Sri Lanka estaba realizando en el Manejo de Costas eran pasos de tipo más tecnocrático.

De estos procesos de diagnóstico surgió la importancia de tratar en el CZMP temas como el sistema regulatorio del CCD (implementación de mejoras en el sistema de permisos), erosión costera, gestión de hábitats costeros, pérdida y degradación de sitios arqueológicos, históricos, culturales, recreacionales y de gran valor paisajístico y, finalmente, se dedicó un capitulo a la revisión del marco legal, administrativo y financiero del sector público en lo que es Manejo de Costas.

\section{- Fase 2. Preparación del Programa}

Los principales objetivos del CZMP manifestados en el Plan fueron los siguientes: Presentar al CCD un plan de trabajo para enfrentar las problemáticas, identificar las acciones a desarrollar por organizaciones gubernamentales y no gubernamentales, así como por el público en general para enfrentar la problemática, y finalmente identificar vacíos de conocimiento en temáticas costeras importantes, para los cuales sea necesario desarrollar investigaciones para llenar esos vacíos.

- Fase 3. Adopción formal y financiamiento

Como mencionamos anteriormente este plan fue encomendado al CCD y a su vez éste lo encomendó a su Planning and Development Branch. De esta manera, para 1990 estaba aprobado el Sri Lanka Coastal Zone Management Plan. La financiación para el desarrollo del primer Plan de Manejo Costero fue en parte responsabilidad del gobierno de Sri Lanka, en parte por la USAID (Coast Conservation Department, 1990).

- Fase 4. Implementación

En cuanto a las acciones referidas para la implementación del Plan frente a cada una de las problemáticas se listó una serie de recomendaciones, pero básicamente todas ellas giraban en torno a acciones de los siguientes tipos: regulación, incluyendo la problemática dentro del sistema de permisos otorgados por el CCD para desarrollar actividades en la ZC; mecanismos de "desarrollo directo" que se refieren al desarrollo de actividades de intervención directamente ejecutadas por órganos gubernamentales: investigación, coordinación, educación y desarrollo de políticas públicas.

- Fase 5. Evaluación

Los éxitos más representativos de la primera etapa de la 51 gestión fueron el aumento de los permisos solicitados para desarrollar actividades en las ZC, la construcción de más de $3000 \mathrm{~m}$ de estructuras para la reducción de la erosión bajo el principio de "Desarrollo Directo" del CCD 
y finalmente la reducción en la explotación y extracción de coral. Por otro lado, se detectaron debilidades, quedando claro que la centralización del proceso y el foco en desarrollo y fortalecimiento institucional no dejaba lugar a aproximaciones participativas que integraran a más niveles de la sociedad, a excepción de interacciones entre científicos y gobierno.

\section{Segunda Generación (1997 - 2004)}

Esta segunda generación de Manejo Costero se basó íntegramente en el primer CZMP. Se trató, en esencia, de una revisión de la primera generación y sobre ella evaluó algunos de los logros alcanzados por la implementación de las acciones planteadas, identificando los aspectos más vulnerables del CZMP, proponiendo nuevas estrategias a seguir e incluyendo nuevos enfoques a la hora de re-diagnosticar las problemáticas costeras.

Es importante destacar aquí el rol de la publicación "Coastal 2000: Recomendations for a Resource Management Strategy for Sri Lanka's Coastal Region" (Olsen et al., 1992), ya que las modificaciones realizadas sobre el primer CZMP son retomadas de esta publicación y del trabajo de investigación que la URI y el CCD desarrollaron conjuntamente sobre las acciones y resultados obtenidos de la primera generación del CZMP.

En lo referido a las observaciones realizadas y adoptadas en Olsen et al. (1992) quedaba claramente establecido que la nueva generación del CZMP debería ser implementada a varios niveles: nacional, provincial, distrital y local, así como también se debería incorporar dentro de estas acciones al sector privado. Se estableció que se debían desarrollar programas de monitoreo con más ímpetu, también se reforzó la idea del fortalecimiento institucional y de los recursos humanos nacionales de Sri Lanka. Se identificó que aspectos primordiales para este nuevo CZMP debían ser la implementación de programas que apunten a generar mayor conciencia pública y educación sobre la costa (Lowry et al., 1999b). También se incorporó la figura de los SAM (Special Area Management Plans), concepto que se manejó desde el comienzo del proceso del CZMP, pero que no había sido incluido. Para el año de la publicación de Olsen et al. (1992) existían cuatro zonas donde ya se implementaban los SAM: Hikkaduwa, Rekawa, Muthurajawela y Negombo Lagoon. Basándose en estas experiencias de los SAM, el segundo CZMP incorpora esta figura y establece procedimientos básicos para su implementación y un listado de zonas especialmente vulnerables donde se podrían y deberían implementar (Coast Conservation Department, 1997). Finalmente, en cuanto a las innovaciones incorporadas a este plan con respecto al anterior, cabe anotar que se reconoce el problema de contaminación y polución de las aguas, se establece una protección de sitios exclusivos y se elaboran planes específicos en los SAM.

\section{Tercera Generación (2004 - Actualidad)}

Al igual que la generación anterior, ésta se basa íntegramente en los logros alcanzados en los dos planes anteriores, estructurando nuevas problemáticas identificadas en las ZC. Se plantearon nuevos objetivos sin dejar de atender a los establecidos por los procesos anteriores, es decir, a través de esta última etapa de procesos acumulativos y dinámicos resultó el CZMP de 2004.

Los principales logros alcanzados y enumerados en el Plan con respecto a sus antecesores fueron los siguientes: mitigación de la erosión en la zona costera, reducción considerable de la extracción de arena y de corales, análisis de impacto ambiental en los casos de actividades de la zona costera, consolidación del sistema de permisos para desarrollar actividades que impacten en la zona costera, incremento de los accesos públicos a la costa, aumentando la conciencia ambiental sobre la problemática costera (se incluyó en las agendas escolares), promoción de la participación local en los procesos de manejo costero e incorporación efectiva de los SAM.

En este Plan se identificaron nuevas problemáticas que no habían sido mencionadas en los planes anteriores, exponiéndose los siguientes: falta de coordinación interinstitucional, falta de capacidad para integrar herramientas de SIG (Sistemas de información Geográfica), falta de fortalecimiento institucional (particularmente y en especial del CCD) y falta de procesos participativos en la elaboración de Planes de Manejo.

Las innovaciones más destacables que lo diferencian de los CZMP anteriores son las siguientes: reconocimiento explícito en el documento de la adopción oficial de técnicas integradas de manejo de recursos naturales (inclusión de comunidades locales en los procesos de manejo y mayor comunicación e integración entre las instituciones actuantes en las $\mathrm{ZC}$ ), reconocimiento explícito a la cooperación internacional que financió las distintas fases de los proyectos de manejo costero e incorporación de un capítulo entero sobre manejo de Pesca y Acuicultura. 


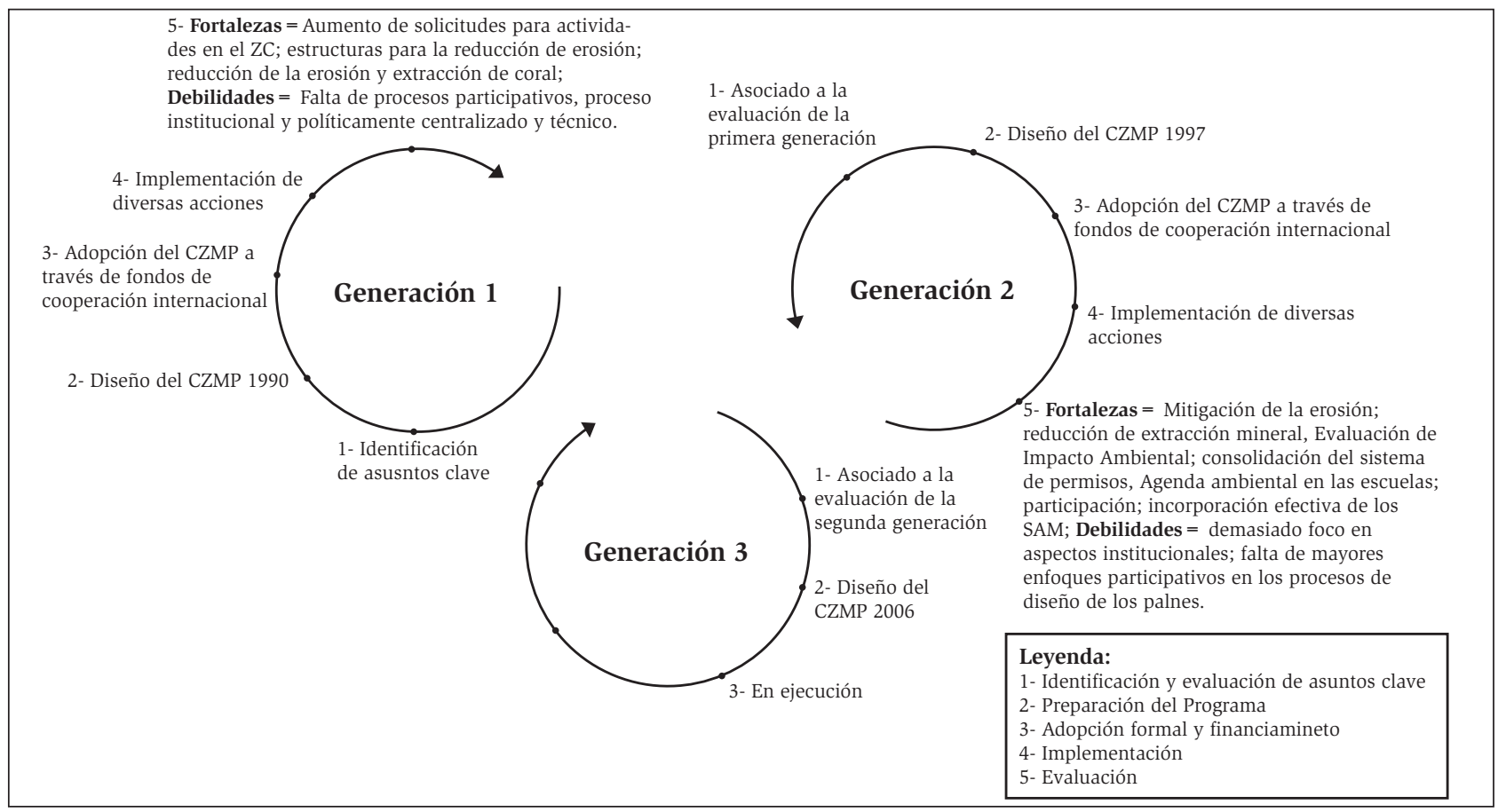

Figura 2. Esquema representando el proceso de Manejo Costero Integrado de Sri Lanka siguiendo el modelo de análisis propuesto por GESAMP (1996). En la figura las siglas CZMP corresponden a Coastal Zone Management Plan; ZC a Zona(s) Costera(s); SAM a las Special Area Management Plans.

\section{DISCUSIÓN}

Lo que en un principio parecieron procesos similares de $\mathrm{MCI}$, por diversas razones resultó en dos procesos claramente diferenciables entre sí. A pesar de compartir un mismo origen en cuanto a la procedencia de los fondos iniciales, la forma de administración de estos fondos y la respuesta a las primeras influencias extranjeras fue totalmente distinta, lo que marcaría a futuro el desenvolvimiento de los procesos. Por un lado, Ecuador detecta su necesidad de desarrollar una iniciativa de Manejo de Recursos Costeros (MRC) a través del "Seminario de ordenación y desarrollo integral de las zonas costeras" y a partir de ese momento, aparentemente el proceso no comenzó sino cuando surgió la oportunidad que planteó la USAID como parte de su proyecto de cooperación internacional. Ecuador no pasó por una etapa de selección y relevamiento de posibles enfoques previamente a la adopción de un modelo y un paquete de cooperación, sino que por el contrario adoptó el modelo y el paquete que le fue ofrecido. Sri Lanka, por el contrario, comenzó su proceso de forma un poco más ordenada, estableciendo primero un marco institucional que sirviera de base al comienzo del proceso de MRC, el Coast Conservation Act N57 (Sri Lanka, 1981) (Lowry y Wickremeratne, 1989;
Lowry y Sadacharan, 1993; Olsen y Christie, 2000). Seguidamente, representantes estatales financiados por el Programa de Naciones Unidas para el Desarrollo parten en busca de posibles modelos y paquetes que pudieran aplicar. Recorren varios países de Europa y también visitan los Estados Unidos, evaluando las opciones y decidiendo adoptar el modelo norteamericano que contenía la cooperación de USAID - URI.

Otro hecho importante es la relación que se mantuvo entre la asistencia técnica y el Estado receptor de la cooperación a posteriori de la firma del Convenio. En Ecuador, la influencia de la URI fue particularmente fuerte; desde el comienzo del proceso se puede observar su clara influencia en el diseño de la agenda de manejo costero, en parte porque dentro del apoyo de esta asistencia técnica se había incorporado en su equipo de trabajo a uno de los principales actores académicos de Ecuador y gestores del proceso, el Dr. Emilio Ochoa. Pero también esta influencia se debe a la debilidad política general en asuntos ambientales de Ecuador en aquel momento. La falta de capacidades técnicas y de recursos humanos en el Estado seguramente aportó a la debilidad política e institucional de Ecuador, que se ve avasallada por una influencia bien formada y de gran ímpetu que llega desde el exterior. 
Por otro lado, Sri Lanka mantuvo una posición un tanto más firme que Ecuador a la hora de manejar los recursos de la cooperación, en especial la asistencia técnica. Un claro ejemplo de esto es la manera en que el Estado de Sri Lanka manejó la propuesta resultante de la primera etapa de la cooperación entre Estados Unidos y su país. En 1990, casi al mismo tiempo que se discutía la aprobación del primer plan de manejo costero en Sri Lanka, culminaba la primera etapa de esta cooperación y se plasmaba el resultado de la misma en la publicación de Olsen et al. (1992) El gobierno de aquél tiempo en Sri Lanka finalmente desestimó la mayor parte de las recomendaciones realizadas y optó por un plan de manejo menos complejo pero que significara menos compromisos económicos para su país. De cualquier manera, estas recomendaciones fueron incorporadas con el tiempo en las siguientes generaciones de MCI.

En cuanto al aspecto institucional, en Sri Lanka es posible encontrar una estructura de trabajo más ordenada, con funciones bien definidas. Desde un comienzo el CZMP estuvo bajo el mandato del Ministerio de Pesca y Recursos Oceánicos y la figura del CCD, como punto de partida, evitó problemas institucionales. Ecuador bajo la figura del PMRC tuvo problemas de coordinación de las distintas instituciones vinculados al proceso. Fue por esta razón que, en el gobierno del presidente Correa, el PMRC fue integrado al Ministerio del Ambiente y supervisado de cerca para aumentar la eficiencia y eficacia de la acción coordinadora de este órgano.

En ambos procesos el espectro de actores involucrados es muy amplio, incorporando actores gubernamentales y no gubernamentales. La diferencia esencial entre los dos enfoques de los países evaluados es que Ecuador ha tenido desde su comienzo un enfoque más participativo y de trabajo a nivel local. Aquí nuevamente se observa la influencia de la asistencia técnica, ya que el elemento participativo fuerte de Ecuador son las ZEM, concepto y práctica que fue sugerido y formaba parte del paquete y modelo norteamericano. De esta manera se intentaba integrar a los actores del sector público a escala nacional a través del primer sendero y a los actores también del sector público pero a escala local conjuntamente con las comunidades locales, las ONGs y el sector privado, lo que según Olsen y Christie (2000) es el mayor desafío 54 del MCI, una gestión de doble vía o la cogestión.

Sri Lanka, por el contrario, comenzó su proceso con un enfoque top-down y sin ninguna participación ni consideración por la escala local, aunque con el paso del tiempo Sri Lanka fue aumentando el nivel de incorporación y adopción de acciones a nivel local y de integración de los distintos actores involucrados. Hacia el final de ambos procesos, se destaca el impulso y la iniciativa de ambos estados de incorporar en todas las etapas del Ciclo de Manejo a la mayor cantidad de actores involucrados, tanto del sector público nacional y local, como del sector privado, ONG's y comunidades locales.

Utilizando el criterio desarrollado por Olsen et al. (2004), encontramos que ambos países han alcanzado resultados de primer orden (cambios institucionales) sustanciales. Los resultados de segundo orden (cambios en el comportamiento) resultan más difíciles de establecer porque se tornan más difusos, aunque Olsen et al. (2004) mencionan algunos de los resultados de segundo orden obtenidos: "La implementación de planes en las ZEM ha producido cambios en el comportamiento de los usuarios de recursos en algunas localidades, tales como menor tala de manglar y mejor manejo de desperdicios sólidos y aguas servidas". Sri Lanka ha logrado este tipo de resultados gracias a que "Se ha desalentado las construcciones, y se ha parado la extracción ilegal de coral y arena. Se han reducido los costosos impactos ambientales provenientes de nuevos desarrollos, y se están construyendo hoteles nuevos en periodos adecuados, reduciendo así el monto público destinado para la onerosa protección costera” (Olsen et al., 2004).

Finalmente, con base en la literatura existente sobre Ecuador y Sri Lanka, se considera que existen casos concretos donde se han alcanzado resultados de tercer orden (cambios socioeconómicos y ambientales). De hecho, Olsen et al., (2004) citan un ejemplo concreto de resultados de tercer orden en Rewaka, Sri Lanka, donde "los residentes mejoraron como resultado de la implementación de un plan de $\mathrm{MCI}$ enfocado en la rehabilitación de la pesca y el desarrollo del turismo". En el caso de Ecuador, a pesar de no ser mencionado explícitamente, de acuerdo al tipo de política de doble sendero que se ha aplicado creemos que se han obtenido resultados de este tipo en algunas localidades.

No se lista resultados de cuarto orden ya que tal y como lo explicitan Olsen et al (2004) "La diferencia entre los resultados de tercer y cuarto orden es que los de tercer son medibles en términos de metas del programa (misión), y los de cuarto son el blanco final que inspira la visión del programa”. 
A través del análisis de ambos proceso se pueden sistematizar una serie de lecciones aprendidas:

1. Procesos Participativos: Primeramente, se destaca la importancia de los procesos participativos a la hora de diseñar e implementar planes de Manejo Costero Integrado. De acuerdo a ambos casos, la participación de actores sociales locales en los procesos de toma de decisión respecto a los temas de manejo mejora significativamente los procesos de implementación de las acciones tomadas.

2. Voluntad Política: También resulta de particular importancia la existencia, con antelación a la aparición de los fondos, de una voluntad política nacional de desarrollar planes de manejo. Como lo demuestra en caso de Sri Lanka, esto permite planificar y buscar fondos que se ajusten a las necesidades del estado que desarrollará estos planes, en lugar de ajustar los planes a las necesidades marcadas por las fuentes de financiación internacionales.

3. Procesos de Evaluación: Los procesos de evaluación de la implementación de cada una de las generaciones de los planes también dieron frutos sumamente importantes, ya que permitieron la constante evolución de los programas, tanto en el monitoreo de sus acciones como en la detección de nuevas problemáticas no identificadas en etapas previas, para diseñar nuevos abordajes en la siguiente generación.

4. Fuentes de Financiación: Las fuentes de financiación internacionales resultan fundamentales en este tipo de procesos, ya sean incipientes o avanzados.

A modo de recomendaciones para futuros procesos de $\mathrm{MCI}$, con base en los casos analizados se plantean las siguientes:

a. Accesibilidad a la información: Un aspecto que no fue enteramente contemplado por ninguno de los dos procesos es el de la sistematización y accesibilidad a la información generada a través de las diferentes etapas de cada ciclo. Si bien la información para el presente trabajo fue relativamente fácil de encontrar, se considera que la forma de diseminar la información podría ser más organizada y sistematizada. Los documentos analizados se encontraban dispersos en varios sitios. Por ello, una centralización de la información y la puesta a disposición a través de recursos virtuales públicos y de fácil acceso generaría mayor transparencia a los procesos y permitiría que un mayor número de personas puedan acceder a ella y así quizás generar aportes mayores a los procesos. La accesibilidad a la información es también una forma de dar mayor legitimidad a los procesos de $\mathrm{MCI}$. b. Programas de Manejo Costero Integrado Regionales: Las causas y consecuencias de muchos de los procesos costeros se expanden más allá de las fronteras nacionales de cada Estado. Una recomendación que se considera conveniente es la de enfatizar esfuerzos en la coordinación regional de Planes de Manejo Costero Integrado. Si se toma el caso de Sri Lanka en particular cabe anotar su participación en lo que se denominó el "Bay of Bengal Large Marine Ecosystem Project". Este proyecto multinacional financiado por la FAO y por el Global Environmental Facility (GEF) integra a 8 países (Maldivia, India, Sri Lanka, Bangladesh, Myanmar, Tailandia, Indonesia y Malasia), para coordinar esfuerzos con el objetivo de generar acciones orientadas a mejorar la calidad de vida de las poblaciones costeras de esta región a través de un enfoque de manejo ecosistémicos de la Bahía del Bengal y de sus pesquerías. Encontramos que este tipo de enfoques sería provechoso en más de un contexto, aunque entendemos que no todos los contextos son los mismos y debe existir una clara voluntad política de todas las partes para colaborar en un emprendimiento regional. Esto no significa que pequeños pasos de coordinación no se puedan llevar a cabo como eventos de capacitación regionales donde se inviten tanto a científicos como a distintos niveles de tomadores de decisiones para capacitarse en temas socio-ecológicos complejos asociados a procesos costeros regionales.

c. Análisis comparativos internacionales: Se encuentra de sumo provecho realizar una investigación que desarrolle un análisis comparativo internacional a mayor escala, con más países y más fuentes de financiación para poder extraer mayores conclusiones, lecciones aprendidas y comparar los distintos procesos. También sería importante en este trabajo establecer el rol en estos procesos de las redes de actores sociales como los científicos, la población local y el sector político. De esta manera se podría generar información respecto a las relaciones entre ellos y el impacto de estas redes en los procesos de implementación de los programas.

En lo que respecta al modelo GESAMP utilizado para el análisis de los procesos, si bien como herramienta teórica el modelo cíclico resulta muy útil para organizar y sistematizar información con el objetivo de llevar a cabo análisis teóricos de procesos de MCI y organizar las fases de Planes de Manejo Costero Integrado, muchas veces la realidad podría chocar contra una herramienta tan rígida. La realidad marca que los procesos políticos no siempre acompañan los procesos técnicos o teóricos, y muchas veces las fases se saltan o evitan por distintas razones. 
Si bien este no fue el caso de ninguno de los dos países analizados, habría que analizar en profundidad otros casos para intentar comprender como los ciclos políticos y la transición entre una administración electa y otra, así como la circulación de personal dentro del Estado puede impactar la fluidez de implementación de estos procesos en el marco teórico de los ciclos del GESAMP. Con base en estos resultados se podría generar una adaptación de este modelo de ciclos que contemple estos aspectos del aterrizaje de este tipo de herramientas teóricas al campo de la realidad.

Otro punto importante es la retroalimentación de información del sistema. Teniendo en cuenta que el modelo propone evaluaciones únicamente en la fase final de cada generación de MCI, se considera de interés integrar evaluaciones al final de cada una de las fases con el objetivo de detectar y solucionar problemas que vayan surgiendo de forma simple y temprana.

\section{CONCLUSIONES}

El análisis comparativo internacional de procesos de manejo costero resulta una forma de estudio muy productiva ya que involucra distintas variables y dinámicas. Se intentó seguir el modelo de las etapas sugerido por GESAMP (1996), pero se encontró que cada país experimentó dinámicas distintas a pesar de presentar en principio muchos aspectos comunes. Esto dificulta el análisis pero enriquece la discusión ya que fortalece la idea de que no existe una receta universal para desarrollar procesos de MCI. Cada sitio posee características sociales, institucionales, culturales, económicas y ambientales distintas que construyen relaciones sociales únicas, las cuales a su vez determinarán de forma diversa y compleja las formas de manejar situaciones, enfrentar los problemas y explotar las potencialidades.

A través de este análisis fue posible detectar que ambos países tuvieron la oportunidad de construir un proceso de Manejo Costero Integrado a partir de la cooperación internacional y lograron que los mismos perduren en el tiempo alcanzando una sostenibilidad financiera y política a través del aprendizaje de los errores y los aciertos acumulados de generación en generación. También se concluye que los procesos participativos no son meramente una herramienta, sino que son parte fundamental de la democracia y pueden generar soluciones para los conflictos existentes en las zonas costeras. Por otro lado, se destaca que el conocimiento tradicional tiene mucho valor y es necesario incorporar a las comunidades en los procesos, ya que resulta la mejor forma de hacerlos sustentables en el tiempo y en el espacio.

Los dos países presentaron muchas debilidades a lo largo de sus respectivos procesos de $\mathrm{MCI}$, de carácter económico, político, organizacional, conceptual y quizás hasta de integración, pero también presentaron fortalezas, principalmente a través de la capacidad de reconocer las dificultades para la implementación de sus respectivos planes de manejo y su incorporación en las generaciones siguientes. Esto se comprobó con el aumento de las temáticas abarcadas con el paso del tiempo. Por un lado, Sri Lanka tuvo mayor iniciativa, al buscar alternativas y distintos procesos antes de aplicar lo elegido. Fue más autónoma en las decisiones y aprovechó mejor los recursos de la cooperación. Por otro lado, Ecuador demostró más dependencia de la cooperación internacional, pero desde el primer Plan de Manejo asumió una postura menos centralizada, optando por la iniciativa de los dos senderos, del nacional para el local y del local para el nacional, lo que permitió concretar las ZEM. Sri Lanka apenas en la tercera generación empezó a tener una postura menos centralizada buscando la integración gobierno/ sociedad/sector privado.

En cuanto a lecciones aprendidas, se puede concluir que los procesos participativos, la voluntad política, los procesos de evaluación y la financiación son fundamentales para el éxito de un proceso de MCI. Sobre recomendaciones, se encuentra relevante incorporar a cualquier plan de manejo aspectos que contemplen accesibilidad a la información. Esto es fundamental para gestionar procesos transparentes, implementación de Proyectos Pilotos e iniciativas de Programas de Manejo Costero Integrado Regionales. Una última recomendación es dirigida a fortalecer la generación de conocimiento sobre procesos de implementación de Planes de Manejo Costero Integrado a través de más investigación internacional comparativa, a fin de extraer lecciones aprendidas y recomendaciones de carácter más universal, si a ello hubiere lugar.

Finalmente, se concluye que el modelo de ciclos de Manejo Costero Integrado planteado por la GESAMP, si bien representa una herramienta teórica fundamental para la planificación y análisis de los procesos de MCI, podría ser revisado y contrapuesto empíricamente 
a través de una investigación más profunda con un mayor número de casos internacionales concretos de implementación de planes de MCI. Esto podría generar una base empírica sobre la cual consolidar o ajustar el modelo de forma tal que sea más flexible y acompañe los a veces dispares tiempos políticos de implementación.

\section{BIBLIOGRAFÍA}

Armada del Ecuador. 1983. Ordenación y desarrollo integral de las zonas costeras: informe del seminario realizado por el gobierno del Ecuador conjuntamente con las Naciones Unidas, Guayaquil, Ecuador, 18-27 de mayo de 1981, 556 pp.

Arriaga, L. 2000. Manejo Costero Integrado (MCI) del Ecuador. Programa de Manejo de Recursos Costeros (PMRC). ESPOL, Guayaquil 129 pp.

Arriaga, L., M. Montano y J. Vasconez. 1999. Integrated management perspectives of the Bahía de Caraquez zone and Chone River estuary, Ecuador. Ocean \& Coastal Management 42: 229-241.

Barragán, J. 2001. The Coasts of Latin America at the End of the Century. Journal of Coastal Research 17(4): 885-899.

Cicin-Sain, B y R.W. Knecht. 1998. Integrated Coastal and Ocean Management: Concepts and Practices. Island Press, Whashington DC, 517 pp.

Coast Conservation Department. 1990. Coastal Zone Management Plan. En: Coastal Resources Management Project of the University of Rhode Island and CCD, Colombo, Sri Lanka, 81 pp. URL: http://www.crc.uri.edu/download/ SLCZMPlan90.pdf Consultado: 05 noviembre 2013.

Coast Conservation Department. 1997. Revised Coastal Zone Management Plan Sri Lanka 1997. Ministry of Fisheries and Aquatic Resources. Colombo, Sri Lanka, 121 pp. URL: www.crc.uri.edu/download/SLCZPlan97.pdf. Consultado: 05 noviembre 2013.

Coast Conservation Departament. 2004. Second Revision of Coastal Zone Management Plan Sri Lanka 2004. Colombo, Sri Lanka, 88pp. URL: http://documents.gov. lk/Extgzt/2006/Pdf/Jan/1429-11/1429-11e.pdf Consultado: 05 noviembre 2013.

Coello, S. 2008. Propuesta de: Política Costera del Ecuador (Costas vivas y saludables para el Desarrollo Nacional). Presidencia de la República del Ecuador Programa de Manejo de Recursos Costeros. 8 pp. URL: http:// simce.ambiente.gob.ec/sites/default/files/documentos/ belen/ Política_ costera_de_Ecuador.pdf Consultado: 23 septiembre 2013.

Cummins, V., O.C Mahony y N. Connolly. 2004. Review of Integrated Coastal Zone Management and Principles of Best Practice. Environmental Research Institute University College Cork, Ireland, 84pp. URL: http://www.heritagecouncil.ie/ fileadmin/user_upload/Publications/Marine/coastal zone review. pdf Consultado: 05 noviembre 2013.

Ecuador. 1989. Decreto ${ }^{0}$ 375. Establécese el Programa de Manejo de Recursos Costeros en el Ecuador. RO 117, del 26 de Enero de 1989.

Ecuador. 1992. Decreto nº 3399. Constitúyese el Programa de Manejo de Recursos Costeros (PMRC) como un organismo adscrito a la Presidencia de la República, descentralizado en el manejo económico y con sede en la ciudad de Guayaquil. RO nº 950, del 4 de Junio de 1992.

Ecuador. 1995. Decreto n ${ }^{2451 . ~ C o n s t i t u ́ y e s e ~ e l ~ P r o g r a m a ~}$ de Manejo de Recursos Costeros (PMRC), como un organismo adscrito a la Presidencia de la República, con sede en la ciudad de Guayaquil. RO n 609, del 11 de Enero de 1995.

Ecuador. 2008. Decreto $n^{0}$ 1254. Suprímase el Programa de Manejo de Recursos Costeros (PMRC), debido a que su existencia ha dejado de ser indispensable para el desarrollo de las áreas costeras del país. Dado en el Palacio Nacional, en Quito, a 12 de agosto del 2008. URL: http:// www.ecocostas.org/images/documentos/1218833738_ DECRETO \% 201254 \%2008-2008.pdf Consultado: 23 septiembre 2013.

Ecuador. 2009. Acuerdo Ministerial ${ }^{0} 025$ de fecha 10 de marzo del 2009, se expide el Reglamento Operativo del Proyecto de Manejo de Recursos Costeros-Etapa II (PMRC II).

GESAMP. 1996. The contributions of science to coastal zone management. Grupo Mixto de expertos sobre los Aspectos Científicos de la Protección del Medio Marino (GESAMP). Roma, FAO. Reports and Studies, GESAMP 61: 66 pp. URL: http://www.fao.org/docrep/meeting/003/ w1639e/w1639e00.htm Consultado: 20 septiembre 2013. GESAMP. 1999. La contribución de la ciencia al manejo costero integrado. Grupo Mixto de expertos sobre los Aspectos Científicos de la Protección del Medio Marino (GESAMP). Roma, FAO Informes y Estudios GESAMP 61: 65 pp. URL: http://www.fao.org/docrep/meeting/003/ w1639s/w1639s00.HTM Consultado: 20 septiembre 2013. 
Gonzáles, R., I. Espejel, J.L Fermán y A. García. 2012. La Administración Costera Integral Ustentable En México: Un Intento Fallido de Manejo Integrado de la Zona Costera. Costas, vol. 1, n.1: 107-121.

Granados, P.A. 2012. Manejo Costero Integrado y Sustentabilidad en Iberoamérica: Un análisis propositivo de políticas públicas en las dos caras atlánticas: España, Portugal, Colombia y Panamá. Editorial académica española, Cádiz, 407 pp.

Hildebrand, L. 2002. Integrated Coastal Management: Lessons Learned and Challenges Ahead. Discussion Document for Management Shared Water/ Coastal Zone Canada 2002 International Conference, Hamilton, Ontario Canada June 23-28, 2002, 1-12 pp.

Knecht, R.W. y J. Archer. 1993. Integration in the US Coastal Management Program. England: Elsevier Science Publishers. Ocean and Coastal Management 21: 183-199.

Lowry, K. y H.J.M. Wickremeratne. 1989. Coastal Area Management in Sri Lanka. Canada. Ocean Yearbook 1: 263-293.

Lowry, K. y D. Sadacharan. 1993. Coastal Management in Sri Lanka. Colombo. Coastal Management in Tropical Asia 1: 1-7.

Lowry, .K., S. Olsen y J. Tobey. 1999a. Donor evaluations of ICM initiatives: what can be learned from them? England: Elsevier Science Publishers. Ocean and Coastal Management 42: 767-789.

Lowry, K., N. Pallewatte y A.P. Dainis. 1999b. Policy-relevant assessment of community-level coastal management projects in Sri Lanka. England: Elsevier Science Publishers. Ocean \& Coastal Management 42: 717-745.

Molina, E. 2000. Manejo de la pesquería de post-larva de camarón, Playas. En: Programa de Manejo de Recursos Costeros (Ed). Guayaquil, Serie Experiences de manejo costero 1: 52 pp. URL: http://simce.ambiente.gob.ec/ sites/default/files/documentos/anny/Manejo \% 20de\% 20 la \% 20Pesquer \% C3 \% ADa \% 20Post \% 20Larva \% 20de \% 20 Camar\%C3\%B3n.pdf Consultado: 23 septiembre 2013.

Ochoa, E. 1995. Manejo costero integrado en Ecuador. En: Programa de Manejo de Recursos Costeros. Guayaquil, $417 \mathrm{pp}$.

Ochoa, E. 2001. Experiencias de gestión integrada de zonas costeras en Ecuador y Centroamérica. En: Conferencia internacional sobre gestión integrada de la zona costera uruguaya del Río de la Plata, Montevideo: 61-69.
Ochoa, E., S.B. Olsen y N. Windevoxhel. 2001. Avances del Manejo Costero Integrado en PROARCA/Costas. Centro de Recursos Costeros de la Universidad de Rhode Island (CRC-URI) Centro Regional para el Manejo de Ecosistemas Costeros-Ecocostas. Guayaquil, Ecuador. 70 pp. URL: www. crc.uri.edu/comm/download/MCI_PROARCA_Full.pdf Consultado: 23 septiembre 2013.

Olsen, S.B. 2000. The common methodology for learning: Ecuador's Pioneering Initiative in Integrated Coastal Management. University of Rhold Island, Rhold Island 33 pp. URL: http://www.crc.uri.edu/download/ EcuadorPioneering_ECU_001D.pdf Consultado: 23 septiembre 2013.

Olsen, S.B. y P. Christie. 2000. What Are We Learning from Tropical Coastal Management Experiences? Taylor \& Francis. Coastal Management 28: 5-18. URL: http://41.215.122.106/dspace/bitstream/0/3773/1/ Olsen \%2520and \%2520Christie.Tropical\%2520ICM.2000. CMJ.pdf . Consultado: 27 septiembre 2013.

Olsen, S.B y E. Ochoa. 2007. El Porqué y el Cómo de una Línea de Base para Gobernanza en los Ecosistemas Costeros. En: ECOCOSTAS - CRC-URI - AVINA LOICZ-. Guayaquil, Ecuador. 31 pp. URL: http://www. procostas.org/cms/docs/porque_y_como_Ochoa-Olsen. pdf Consultado: 27 septiembre 2013.

Olsen, S. B., D. Robadue Jr y L. Arriaga. 1995. Un enfoque participativo y adaptativo para el Manejo Costero Integrado. Pp 29-64. En: E. Ochoa (Ed). Ecuador. En: Manejo Costero Integrado en Ecuador; PMRC, CRC-URI, USAID; Documento. 2. 74 p.

Olsen, S.B., D. Sadacharan, J.I. Samarakoon, A.T. White, H.J.M. Wickremeratne, y M.S. Wijeratne (Ed). 1992. Coastal 2000: Recommendations for A Resource Management Strategy for Sri Lanka's Coastal Region, Volumes I and II. CRC Technical Report N²033, Coast Conservation Department, Coastal Resources Management Project, Sri Lanka and Coastal Resources Center, The University of Rhode Island. URL: http://www.crc.uri.edu/download/ SLCoast2000II.pdf Consultado: 05 noviembre 2013.

Olsen, S.B., J. Tobey y L. Hale. 2004. "Qué Estamos Aprendiendo En El Manejo Costero Integrado?" EcoCostas. URL: http://www.ecocostas.org Consultado: 27 septiembre 2013.

Olsen, S.B., J. Tobey y M. Kerr. 1997. A Common framework for learning from ICM experience. Ocean \& Coastal Management 37(2): 155-174. 
ONU. 1972. Declaración de Estocolmo sobre el Medio Ambiente Humano. URL: www.mma.gov.br/estruturas/ agenda21/_arquivos/estocolmo.doc Consultado: 24 septiembre 2013.

ONU. 1982. Convención de las Naciones Unidas sobre el Derecho de Mar. URL http://www.un.org/depts/los/ convention_agreements/texts/unclos/convemar_es.pdf Consultado 24 de septiembre 2013.

PNUMA. 1992. Programa 21. Capítulo 17: Protección de los océanos y de los mares de todo tipo, incluidos los mares cerrados y semicerrados, y de las zonas costeras, y protección, utilización racional y desarrollo de sus recursos vivos. Informe de la Conferencia de las Naciones Unidas sobre el Medio Ambiente y el Desarrollo. (Rio de Janeiro, 3 a 14 de junio de 1992). Programa de las Naciones Unidas para el Medio Ambiente. 191-195 p.

Polette, M. y L. P. Silva. 2003. GESAMP, ICAM e PNGC - Análise comparativa entre as metodologias de gerenciamento costeiro integrado. Ciencia e Cultura [online] 55(4): 27-31. URL: http://cienciaecultura.bvs.br/scielo. php? script $=$ sci_arttext $\&$ pid $=$ S0009-67252003000400017 Consultado: 23 septiembre 2013

Rodríguez, J.J. y N.J. Windevoxhel. 1998. Análisis regional de la situación de la zona marina costera centroamericana, Banco Interamericano de Desarrollo, Washington D.C., No. ENV-121, 103 pp.

Savundranayagam, T., L. de Alwis, L. Joseph y N. Siripala. 1994. The Economic Significance of the Coastal Region of
Sri Lanka. Coastal Resources Center of the University of Rhode Island. 41pp. URL: http://pdf.usaid.gov/pdf_docs/ PNABZ850.pdf . Consultado: 23 septiembre 2013.

Sorensen, J. 1993. The International Proliferation of Integrated coastal zone management efforts. Ocean \& Coastal Management 21: 45-80.

Sri Lanka. 1981. Coast Conservation Act No. 57 of 1981. URL: http://www.commonlii.org/lk/legis/num_act/ cca57o1981263/ Consultado: 05 noviembre 2013.

Stojanovic, T., R.C. Ballinger y C.S. Lalwani. 2004. Successful integrated coastal management: measuring it with research and contributing to wise practice. Ocean and Coastal Management 47: 273-298.

Tagliani, P.R. y M. Asmus. 2011. O Programa de Manejo Integrado do Estuário da Lagoa dos Patos. pp. 27-39. En: Tagliani, P.R y M. Asmus (Ed.). Manejo Integrado do Estuário da Lagoa dos Patos: uma experiência de gerenciamento costeiro no sul do Brasil. Editora da FURG, Rio Grande. 252 p.

Vallega, A. 1999. Fundamentals of Integrated Coastal Management, Dordrecht, Kluewer Academic Publishers, $288 \mathrm{pp}$.

World Bank. 1993. The Noordwijk guidelines for integrated coastal zone management. Paper presented at the World Coast Conference, 1-5 November 1993, Noordwijk. The Netherlands. Washington DC. The World Bank, Environment Department, $21 \mathrm{p}$.

Fecha de Recepción: 30/08/2014

Fecha de Aceptación: 02/10/2014

Para citar este artículo: Avellar-Mascarello, M., M. García-Cartagena y E. Jara-Torres. 2014. Procesos de manejo costero integrado en Ecuador y Sri Lanka: una perspectiva de comparación. Revista Intrópica 9: 43 - 59 